\title{
Identidad y dignidad humanas versus mejora humana no-terapéutica
}

\author{
Human identity and human dignity \\ versus non-therapeutic human enhancement
}

\author{
JESÚS PARRA SÁEZ \\ Universidad de Murcia
}

Recibido: 21/09/18 Aceptado: 09/10/18

\begin{abstract}
RESUMEN:
En el debate científico-ético contemporáneo en torno a la mejora humana biotecnológica, las nociones de identidad y dignidad humanas son utilizadas de forma habitual por parte del sector de pensamiento más conservador, para rechazar la aplicación de las nuevas tecnologías biomédicas sobre el ser humano con el objetivo de llevar a cabo una mejora de carácter noterapéutico. Sin embargo, ¿sirven éstas como argumento para rechazar una mejora humana de carácter perfeccionador? En este artículo se analizará el significado de ambas nociones en el debate bioético contemporáneo, así como la validez de su uso como argumento para rechazarla.
\end{abstract}

PALABRAS CLAVE

MEJORA HUMANA, DIGNIDAD, IDENTIDAD, BIOCONSERVACIONISMO, BIOTECNOLOGÍA

\section{ABSTRACT:}

In the contemporary scientific-ethical debate on biotechnological human enhancement, the notions of human identity and human dignity are used habitually by the most conservative line of thought to reject the application of new biomedical technologies on the human being with the aim of carrying out an improvement of a non-therapeutic nature. However, are they

(C) Contrastes. Revista Internacional de Filosofía, vol. XXIV-Nº2 (2019), pp. 7-25. ISSN: 1136-4076

Departamento de Filosofía, Universidad de Málaga, Facultad de Filosofía y Letras Campus de Teatinos, E-29071 Málaga (España) 
useful as an argument to reject a human enhancement of a perfectionist nature? In this article, we will analyze the meaning of both notions in the contemporary bioethical debate, as well as the validity of their use as an argument to reject it.

KEYWORDS

HUMAN ENHANCEMENT, DIGNITY, IDENTITY, BIOTECHNOLOGY, BIOCONSERVATISM

El PERFECCIONAMIENTO HUMANo ha sido una constante desde la aparición de los primeros Homo Sapiens, y gracias al desarrollo de las civilizaciones humanas y de la techné ha sido posible materializar una mejora cualitativa y cuantitativa de nuestras habilidades naturales, y de nuestra calidad de vida. El humanismo renacentista, el movimiento ilustrado y la revolución industrial nos han llevado al culmen de la evolución humana, tanto a nivel biológico como a nivel social y cultural. Sin embargo, esta forma de perfeccionamiento humano «ambiental» o «natural» ha dado paso a un tipo de mejora artificial fundamentada en la aplicación de derivados químico-farmacológicos y artefactos tecnológicos (revolución de las tecnologías biomédicas) que promete llevar a cabo una mejora humana radical (la consecución de un nuevo Prometeo) a lo largo de este siglo. En este prometedor y, al mismo tiempo, inquietante escenario anticipado por las denominadas utopías transhumanistas, el sector científico y filosófico de pensamiento más conservador -habitualmente conocido como bioconservacionismo-acude a las nociones de «identidad humana» $\mathrm{y}$ «dignidad humana» para rechazar cualquier tipo de intervención artificial-especialmente biotecnológica- cuyo fin no sea exclusivamente terapéutico (detección y eliminación de patologías). En este artículo se analizarán las nociones de identidad y dignidad humanas, y su significado en el debate bioético contemporáneo en torno a la legitimidad de la aplicación de biotecnología sobre el ser humano; las consecuencias de una mejora humana de carácter no-terapéutico para ellas; y la validez de su uso con el objetivo de rechazar un perfeccionamiento artificial de naturaleza perfeccionadora, es decir, la mejora o extensión de características no relacionadas directamente con la cura de enfermedades como la potencia física, la inteligencia y la capacidad moral.

\section{IDENTIDAD Y DIGNIDAD HUMANAS. ¿A QUÉ ALUDEN?}

La identidad humana ha sido definida a lo largo de los siglos -en consonancia con los acontecimientos históricos y la emergencia o desaparición de procesos y movimientos científico-sociales- de muchas formas distintas (Cantarino 2015). Algunas de éstas apuntan hacia un fundamento metafísico y ontológico de aquélla, mientras que otras hacen lo propio hacia un sentido más personal y cultural relativo a un fundamento psicológico y, en ocasiones, espiritual o sentimental. Sin ir más lejos, los primeros factores distintivos de 
un sujeto son su estructura físico-biológico-genética (distinta a la del resto de sujetos) y su estructura psicológico-mental (estados mentales individuales), aunque las definiciones de identidad como cuerpo y cerebro o como mente y cognición son habitualmente rechazadas por parte del ámbito científicofilosófico por su reduccionismo. Tal y como se pregunta Parfit, «¿consiste la identidad personal en la continuidad corporal y psicológica, o es un hecho más, independiente de los hechos que recogen estas continuidades?» (Parfit 1983, p. 36). Desde el punto de vista teológico se señala al alma, a la moralidad, y al obrar del sujeto como factores indispensables de la identidad humana, mientras que desde el punto de vista jurídico, el derecho nacional e internacional ha tratado de agrupar estos tres puntos de vista en el establecimiento de la identidad como derecho humano fundamental. De este modo, constituciones como la española establecen que la identidad humana es un atributo esencial de cada sujeto que le otorga el derecho a ser reconocido por cómo es (física, intelectual y moralmente), mientras que el derecho internacional -en declaraciones y tratados como la Declaración Universal de los Derechos Humanos y la Convención sobre los Derechos del Niño- establece, también, la dimensión social, cultural y jurídica de la identidad personal única e intransferible de un sujeto que lo distingue del resto.

Asimismo, la cuestión del «yo», el reconocimiento o consciencia de sí mismo y la identidad personal, cultural y colectiva han sido una constante en la historia de la filosofía y del derecho (Laporta 2013), siendo la noción lockeana sobre la continuidad psicológica (memorística) del sujeto en Ensayo sobre el entendimiento humano, la idea de identidad (yo) como cierto hecho psicológico que Hume intenta encontrar en Tratado sobre la naturaleza humana, y el «cogito» cartesiano en El discurso del método algunas de las propuestas más conocidas (Cincunegui 2015). Es cierto que la incertidumbre acerca de a qué nos estamos refiriendo cuando hablamos de identidad humana complica bastante la empresa de establecer cuáles podrían ser las consecuencias de las aplicaciones biotecnológicas sobre el ser humano para ella. No obstante, la diferenciación establecida por Derek Parfit en el seno de su obra Razones y personas de 1984 (Parfit 2004) abrió la puerta a un cierto consenso acerca de qué entendemos por «identidad personal» en los ámbitos de la filosofía y la bioética, y en la problemática de la identidad en relación a los avances científicotecnológicos. En dicha obra, Parfit distingue entre «identidad cualitativa» e «identidad numérica», de modo que dos sujetos pueden ser cualitativamente idénticos (seres física y psicológicamente idénticos) pero numéricamente distintos (ontológicamente no son el mismo sujeto, hay dos sujetos distintos). Al mismo tiempo, si un ser humano cambia su «forma de ser», esto es, su carácter o personalidad, será numéricamente idéntico a sí mismo (es el mismo sujeto) pero cualitativamente distinto (no tiene exactamente la misma personalidad, 
carácter o características físicas). En última instancia, para Parfit la identidad numérica alude a aquello que «necesariamente implica o a aquello en lo que consiste» (Parfit 2004, p. 383), tanto a nivel físico como psicológico-mental; mientras que la identidad cualitativa alude al contenido de la vida de una persona, esto es, sus pensamientos, deseos, intenciones, experiencias, etc.

Con esta distinción entre identidad cualitativa y numérica establecida por el pensador británico como base, en el debate contemporáneo en torno al mejoramiento humano biotecnológico se habla, en general, de dos formas de identidad: numérica y narrativa (DeGrazia 2005; Salles 2015). Grosso modo y en consonancia con Parfit, la «identidad numérica» alude a aquello que necesita ésta para seguir existiendo a través del tiempo, guardando en sí un plano biológico y un plano psicológico. Desde el primero la identidad numérica señala a la permanencia de la estructura biológica del individuo (supervivencia corporal), mientras que desde el segundo se señala a la permanencia de la capacidad mental del individuo (supervivencia mental). Por el contrario, y en lo que toca a la «identidad narrativa», ésta alude a todas aquellas características psicológicas y sentimentales que definen a un sujeto como tal (único) y lo diferencian del resto de sujetos, es decir, el contenido de la vida personal de un individuo. Habitualmente estas características son identificadas con las vivencias que son exclusivas del sujeto, a saber: creencias, experiencias, recuerdos, deseos e intereses personales. En la medida que la supervivencia físico-mental del sujeto es precisa para que se dé la identidad narrativa, ésta es dependiente de la identidad numérica.

Parece evidente que, en lo que respecta a la identidad numérica en su sentido más biológico-esencialista, sólo un cambio manifiestamente radical en la estructura físico-genética del individuo podría provocar la pérdida o eliminación de su identidad (muerte biológica, inexistencia). Aquí cabría debatir en torno a la cada vez menos pretendida mejora física (perfeccionamiento de características como la resistencia o el crecimiento muscular) y la cada vez más esperanzadora cibernética (sustitución de miembros u órganos por análogos artificiales cuya funcionalidad iguala o supera con creces lo meramente natural). Al mismo tiempo y de forma similar, las capacidades cognitivo-mentales del individuo se verían afectadas debido a una alteración manifiesta de nuestro cerebro y nuestra mente. En este sentido, cabría hablar del tan ansiado mejoramiento cognitivo (incremento de las capacidades cognitivas de asimilación de datos, respuesta mental e inteligencia), y aún más de la denominada transferencia mental (conversión de nuestra mente en datos informáticos y transferencia de éstos a un habitáculo artificial). No obstante y como bien observa Salles, respecto a las intervenciones artificiales de perfeccionamiento: 
Lo que causa mayor ansiedad no es que las personas pierdan su identidad numérica dado que esta cirugía no cambia a los pacientes de modo tan radical que deje de existir la continuidad psicológica o física relevante. Más bien, la inquietud sobre la identidad personal responde a la posibilidad de que cambios en el humor, el carácter y hasta ciertas alteraciones cognitivas modifiquen quienes los pacientes «verdaderamente son», es decir, su forma de vida psicológica (Salles 2015, p. 61).

Así, de forma habitual en el debate científico-filosófico contemporáneo en torno al mejoramiento humano biotecnológico se alude al aspecto más psicológico y sentimental de la identidad humana, y por tanto, la identidad narrativa es el objeto utilizado a la hora de rechazar el perfeccionamiento no-terapéutico. Más tarde, veremos como las posibles consecuencias de la mejora humana perfeccionadora para la identidad se centran en la pérdida de esta identidad narrativa.

En cuanto a la dignidad humana, es un hecho que, en lo que al perfeccionamiento humano se refiere, buena parte de los ámbitos ético-filosóficos rechazan una mejora no-terapéutica «en la medida en que alienta el que los intereses de la ciencia y de la sociedad estén por encima de la dignidad humana y del principio de que el fin no justifica los medios» (Laing 2009, p. 65). Ahora bien, ¿a qué aluden estos pensadores cuando acuden al término «dignidad humana»? El derecho internacional ha utilizado este término en diversas ocasiones, aunque sin un sentido unívoco. La Declaración Universal de los Derechos Humanos de 1948 habla de «dignidad intrínseca» de todos los miembros de la especie humana en tanto que pertenecientes a ésta. En el Convenio para la Protección de los Derechos Humanos y la Dignidad del Ser Humano con respecto a las Aplicaciones de la Biología y la Medicina de 1997, se habla de respetar al ser humano como persona y como ser perteneciente a la especie humana para reconocer la importancia de la «dignidad humana». Por su parte, la Declaración Universal sobre el Genoma Humano y los Derechos Humanos emitida por la UNESCO en 1997, habla de «dignidad intrínseca» en relación con el genoma humano, los derechos humanos y la diversidad humana. Finalmente, la Declaración Bioética de Gijón del año 2000 situaba a la «dignidad humana» como límite jurídico-moral para la investigación y experimentación científica sobre seres humanos. Debido a esta ambigüedad, existe un amplio debate -especialmente en bioética y filosofía- en torno al significado de este término (Kass 2004 y 2008; Bostrom 2008), tanto a nivel ontológico como moral (¿es el ser humano digno por necesidad al formar parte de la especie humana o lo es en función de su quehacer social y moral? ${ }^{1}$ ). Dos

1 Según Andorno (1998), los seres humanos tenemos una dignidad ontológica (propia de todo ser humano en tanto que ser humano) y una dignidad ética (otorgada a los sujetos en función de su quehacer socio-moral). Del mismo modo, desde el ámbito teológico se ha mos- 
buenos ejemplos de esta ambigüedad, son la dignidad entendida en sentido personalista y la dignidad entendida en sentido utilitarista (Aparisi 2013, Cabrera 2017). Según la primera, la dignidad es una cualidad intrínseca y única de la persona (autónoma, autoconsciente, racional). Lo importante aquí es tener en cuenta que, según esta concepción, no todos los seres humanos son dignos, sino sólo aquéllos que poseen dichas cualidades, es decir, las personas. ${ }^{2} \mathrm{De}$ este modo, la dignidad en sentido personalista aboga por la no unidad del ser humano, y tal como muestra Parfit en Razones y personas, le otorga un valor considerablemente mayor a las personas que a los seres humanos:

La mayoría de nosotros no distingue entre personas y seres humanos (...) Si hacemos esta distinción, podemos llegar a pensar que, mientras que es malo matar a un ser humano, es peor matar a una persona. Podemos incluso llegar a pensar que sólo es incorrecto matar a personas (Parfit 2004, pp. 561-562).

El problema es, por tanto, qué hay de aquéllos sujetos humanos-como los recién nacidos, los que padecen enfermedades mentales o neurológicas severas, e incluso los niños en estado fetal-que no son capaces de ejercer autonomía o racionalidad, ¿no son dignos? ${ }^{3}$ Por su parte, según la concepción de la dignidad en sentido utilitarista, la vida de cualquier ser humano cuya calidad no es óptima no merece la pena ser vivida, y por consiguiente, carece de dignidad. Esta es una concepción de dignidad que ha guardado cierta polémica social en las últimas décadas, especialmente en torno a la legitimización de la eutanasia o del aborto eugenésico. Sin embargo, este sentido de dignidad es tan excluyente como el anterior, pues afirma que cualquier sujeto cuya «calidad de vida» descienda de lo meramente convencional (un ser humano sin problemas biológico-sociales graves) con motivo de un accidente o enfermedad, es indigno. Entonces, toda vida que no sea estrictamente convencional (en sentido de «calidad de vida») ¿no merece la pena ser vivida?, ¿carecen de dignidad los seres humanos susceptibles de vivir tal situación? La mayor parte del entorno bioético contemporáneo, al menos aquellos que se alejan de la ideología posthumanista, rechaza la adopción de estas concepciones de dignidad. Por ejemplo, la catedrática de Filosofía del Derecho Ángela Aparisi entiende que la noción de dignidad humana propone

trado una distinción entre la dignidad intrínseco-ontológica (el ser es digno per se) y la dignidad extrínseco-moral (el ser es digno o indigno en función de su actividad moral).

2 Para pensadores como Peter Singer, la calificación de persona puede ser otorgada indistintamente a seres no pertenecientes a la comunidad humana, como por ejemplo algunos animales. Del mismo modo, cabría la posibilidad de que un ser humano no fuera identificado como persona.

3 Si la respuesta a esta pregunta es negativa, la utilización de embriones, niños en estado prenatal, y personas con discapacidad mental severa para experimentación biotecnológica -por ejemplo la relativa al perfeccionamiento humano- sería aceptable. Sin embargo, tal y como hemos recogido, el derecho internacional rechaza tal concepción de dignidad. 
adoptar la dignidad en sentido ontológico, esto es, como la «calidad de ser» de todas las personas que rechaza la utilización de éstas como meras herramientas y objetos, un respeto de la persona como un todo:

Dignidad es un término que se aplica al hombre para señalar una peculiar calidad de ser, para sostener que es persona y no sólo individuo [...] Lo digno no es sólo su razón o su capacidad de auto-determinarse moralmente, sino también su naturaleza corporal, toda ella penetrada de racionalidad (Aparisi 2013, pp. 207-209).

Esta noción se acerca mucho a la que ha sido ampliamente aceptada por parte del ámbito ético-científico, y es que dentro de la ambigüedad terminológica hay cierto consenso en áreas como la de la ética aplicada a las nuevas tecnologías biomédicas en identificar el término «dignidad humana» con el reconocimiento del ser humano (de su valor) en sí mismo, que establece una dimensión de igualdad y respeto entre seres humanos «con independencia de sus circunstancias personales y sociales, de sus capacidades físicas o mentales y de su propia conducta» (Romeo Casabona 1994, p. 44). El filósofo alemán Jürgen Habermas (2002) es uno de los principales defensores contemporáneos de la dignidad humana en este sentido relacional frente al perfeccionamiento biotecnológico, proponiendo la denominada «ética de la especie»-la autocompresión del sujeto como miembro de la especie humana y el respeto de ésta a través de la simetría en las relaciones morales y sociales que sus componentes mantienen entre sí- como principio ético a seguir para salvaguardarla. De forma similar, la filósofa mexicana Juliana González ha señalado que la dignidad humana «es ciertamente una manera de comprender al hombre, de la cual deriva una manera de «tratar al hombre»; y coincide con la fundamental e inquebrantable forma en que el ser humano ha de verse y asumirse a sí mismo y ha de ver y asumir a los otros seres humanos» (González 2005, p. 65). De este modo, tanto la noción de «dignidad humana» manifestada por Habermas como la expresada por González, ponen su foco de atención sobre la idea de respeto y reconocimiento (recíproco) del yo para el otro y del otro para el yo. Esta idea de dignidad se remonta al concepto estoico de humanitas en el siglo V a. C. (Spinella 2012), aunque también puede rastrearse en Sobre la invención de la retórica de Cicerón, en Sobre la dignidad del hombre de Pico della Mirandola y, posteriormente, en la Metafísica de las costumbres de Immanuel Kant. Éste, como implacable defensor de la dignidad humana, hizo hincapié en la consideración de los seres humanos como fines en sí mismos y no como medios:

La humanidad misma es una dignidad; porque el hombre no puede ser utilizado únicamente como medio por ningún hombre (ni por otros, ni siquiera por sí mis- 
mo), sino siempre a la vez como fin, y en esto consiste precisamente su dignidad (Kant, 2008, p. 335).

De este modo, lo que ya anticipaba Kant antes que Habermas es la relción entre la dignidad humana y el respeto hacia el otro que fundamenta, como veremos a continuación, gran parte del rechazo del entorno bioconservador hacia la mejora humana perfeccionadora.

\section{CONSECUENCIAS DE LA MEJORA HUMANA NO-TERAPÉUTICA PARA AMBAS}

En lo que se refiere a la identidad humana, la denominada identidad narrativa es lo que de forma habitual entendemos por personalidad o carácter, esto es, el conglomerado de intereses, deseos, experiencias, recuerdos que en última instancia nos hace «ser» o, quizás mejor, comportarnos y actuar de una manera u otra. Las corrientes de pensamiento más conservadoras ven en la mejora humana perfeccionadora una verdadera amenaza para ésta, y por ello se erige como uno de los problemas principales del debate científico-filosófico contemporáneo en torno a la legitimidad o no del perfeccionamiento no-terapéutico. A este respecto, Walter Glannon (2002) afirma que la «memoria episódica» humana -la continuidad psicológica de nuestros recuerdos y estados mentales que Locke había adelantado siglos atrás como clave en la supervivencia de nuestra identidad-se vería gravemente afectada por una mejora perfeccionadora radical, como por ejemplo la extensión considerable de nuestra vida (un incremento de cientos e incluso miles de años). Para él, en algún punto de la prolongación de la vida humana nuestras conexiones psicológicas se derrumbarían (asimetría entre la expansión de nuestra vida y la capacidad de nuestra mente/memoria para procesar toda la información con el paso del tiempo), en la medida que las conexiones o experiencias pasadas que nos hicieron «ser» (personalidad y comportamiento) de una forma determinada desaparecerían con el paso del tiempo (desapareciendo con ellas nuestros intereses, deseos e inquietudes):

Las conexiones entre los estados mentales anteriores y posteriores en una vida de aproximadamente 200 años serían tan débiles que no habría ninguna buena razón para preocuparse por los «yoes» que tenían estos estados (...) es poco probable que la identidad de uno y la de la misma persona [en el futuro] se conservaría, debido a la conexión psicológica disminuida durante un período de tiempo muy largo (Glannon 2002, pp. 268-276).

Más recientemente y en el mismo sentido, el filósofo neozelandés Nicholas Agar (2014) ha emitido su preocupación por la afección de este tipo de mejora a la «memoria autobiográfica» del sujeto, esto es, a todas las conexiones psicológicas y experiencias vividas por el sujeto desde su nacimiento (identidad 
narrativa) que lo llevan a obrar y pensar de una manera determinada y que sobreviven -salvo padecimiento de enfermedades que afecten a la mente- hasta el día de nuestra muerte. Para él, aunque el sujeto fuera perfeccionado de modo que no perdiera sus «recuerdos autobiográficos», su «yo» post-mejora tendría poco o ningún interés en quién era él antes de la intervención. Las conexiones psicológicas de su «yo» anterior no se corresponderían con las habilidades y capacidades de las que dispondría después de la mejora y, por tanto, ya no serían centrales para su nueva vida. Así, el sujeto post-mejora enfocaría su vida hacia el futuro, crearía nuevas conexiones psicológicas, nuevos recuerdos y experiencias, y en última instancia, una nueva identidad que nada tendría que ver con la anterior.

Otra posibilidad íntimamente relacionada con la identidad es la de la pérdida de «autenticidad»o afección a la «responsabilidad». Esta amenaza es recogida por el filósofo-político norteamericano Michael Sandel (2015), entre otros, para aludir a la desaparición del sentimiento de autenticidad y responsabilidad inherente a los sujetos humanos cuando obran o logran algún objetivo:

Un aspecto de nuestra humanidad que podría resultar amenazado por la ingeniería genética y el perfeccionamiento es nuestra capacidad (...) para considerarnos a nosotros mismos responsables por nuestra forma de ser y de hacer las cosas (Sandel 2015, p. 69).

La idea de fondo es que un sujeto que ha sufrido un perfeccionamiento de sus habilidades no se hará responsable de sus actos, en la medida que éstos derivan de una serie de capacidades que no son suyas per se, sino que le han sido dadas artificialmente. Más allá del perfeccionamiento genético, este problema alude también en gran medida a la posibilidad de escoger características de un sujeto en su etapa de embrión (selección embrionaria). De este modo, un sujeto que tiene una serie de características físico-cognitivas determinadas (por ejemplo ser más alto, fuerte e inteligente de lo meramente convencional) como consecuencia del interés y elección de sus padres (niño a la carta), podría entender que actúa y «es» de un modo concreto debido a una intervención artificial, pues de forma natural sería de otra manera: «la asunción de lo que somos implicará tener que asumir lo que los otros han hecho; el adolescente ya no dirá: 'soy así porque sí', sino porque otro quiso (o se equivocó al querer) que yo fuera así» (Sagols 2010, p. 32). Así, un sujeto que ha sido perfeccionado con el objetivo de ser un atleta de élite no se sentirá realizado ni responsable con la adquisición de tal grado o estatus, no al menos como lo estaría un sujeto natural que ha logrado el mismo objetivo con esfuerzo y perseverancia. Aquí, tanto el sujeto como sus cualidades (obtenidas artificialmente) son mostradas como una falsificación de lo que hubieran sido de forma natural (inautenticidad). 
Por otra parte y en lo que respecta a la dignidad humana, de forma habitual se señala a técnicas biomédicas como la ingeniería genética (manipulación del genoma humano y de la línea germinal ${ }^{4}$ ), el diagnóstico prenatal y el diagnóstico pre-implantatorio como formas de afección. En torno a éstas, ha emergido un extenso debate acerca de la legitimidad o no de los padres para seleccionar embriones en función de sus características genéticas, esto es, llevar a cabo una «selección eugenésica»:

¿Puede suprimirse a ciertos seres humanos, por el sólo hecho de ser portadores de determinadas enfermedades?, ¿quién tiene derecho a decidir cuáles son los «buenos» genes, que deben ser alentados, y cuáles son los «malos», que justifican la eliminación de su portador? (Postigo Solana y Díaz de Terán, 2004, p. 84).

Así, algunos de los pensadores bioconservadores más conocidos -Habermas, Kass y Annas- afirman que técnicas como la selección embrionaria o la ingeniería genética podrían afectar gravemente la dignidad humana, en la medida que «rompen» con el reconocimiento y respeto recíproco entre sujetos humanos, en este caso, entre los padres (que alteran la estructura genéticohereditaria de sus hijos por intereses personales de forma irreversible) y el futuro niño (al que no se ha respetado y ha sido instrumentalizado). Según esta idea, la autonomía y libertad de elección del sujeto debe ser respetada a rajatabla, por lo que cualquier tipo de intervención perfeccionadora que implique la alteración de sujetos incapaces de manifestar su conformidad con ésta (por ejemplo los sujetos que aún no han nacido) no debe ser permitida. En caso contrario, la dignidad humana tal y como ha sido definida aquí se vería afectada. Con esta idea en mente, pensadores como Victoria Camps (2002) señalan al principio kantiano del imperativo categórico para afirmar que no hay que cosificar a otros seres humanos por intereses propios, ni aunque pudiéramos pensar que es lo mejor para ellos: «no manipules al otro en tu propio beneficio, pues el otro es un ser humano que merece siempre el respeto debido a cualquier otro ser humano. Respetar la dignidad del otro implica no tratarlo como si fuera una cosa al servicio de los propios intereses» (Camps 2002, p. 60). Y es que, como vislumbra Bellver (2012), si los padres decidieran - por la razón que fuese- las características de sus hijos, les estarían imponiendo y sometiendo a un ideal

4 En lo que respecta a la manipulación genética de la línea germinal (efectos perdurables en las generaciones venideras), pensadores como de Miguel (2018a, 2018b) afirman que el uso de la ingeniería genética en sentido terapéutico podría hacer honor a la protección de dicha dignidad. La cuestión de fondo es, ¿qué problema hay con modificar el genoma humano si con ello se consigue eliminar patologías genético-hereditarias graves tanto en el sujeto como en sus descendientes? 
de posthumanidad no escogido libremente por ellos, y por tanto, no estarían respetando su dignidad.

Leon Kass, por su parte, dedica gran parte de su obra Life, Liberty and the Defense of Dignity (2004) a defender la protección de la dignidad humana entendida como lo que importa de la «vida humana real», es decir, el día a día (deseos, pasiones, sentimientos, aversiones, gustos, etc.) frente a la intromisión y posible impacto de tecnologías como la ingeniería genética o la clonación sobre nuestras vidas que Aldoux Huxley había adelantado en su novela Brave New World. Posteriormente, en el seno del Consejo de Bioética del Presidente, Kass (2008) hablaría sobre la protección de la dignidad humana ${ }^{5}$ en su sentido más básico (la que pertenece al ser humano en tanto que ser humano), y en su sentido más complejo (la perteneciente al ser humano en tanto que es y actúa activamente como un ser humano). En esta concepción, la dignidad básica parece actuar como análoga de la identidad numérica (atributos necesarios del individuo que lo hacen un ser digno), mientras que la dignidad plena parece hacer lo propio con la identidad narrativa (atributos morales, sociales y culturales que hacen a los individuos más o menos dignos, o tal vez, honorables). En este sentido, afirma el pensador estadounidense, la primera se vería afectada por acciones que desemboquen o puedan desembocar en la muerte involuntaria (por ejemplo el asesinato, o la esclavitud), mientras que la segunda se vería afectada por las numerosas mejoras perfeccionadoras pretendidas por los posthumanistas (perfección corporal, mental y moral artificial). Una mejora biotecnológica radical podría acabar con nuestra vida, en el peor de los casos, o convertirnos en seres deshumanizados desprovistos de dichos atributos morales y sociales. Por tanto, Kass aboga por proteger ambas: «podemos, y debemos, defender tanto la dignidad del ser humano como la dignidad de ser humano» (Kass 2008, p. 306). Así, no se trataría únicamente de salvaguardar nuestra dignidad básica (libertad, autonomía, igualdad, respeto, derechos), sino también nuestra dignidad en sentido más amplio (nuestros sentimientos, intereses y deseos; nuestra generosidad, moderación y relación con los otros; lo que en definitiva nos hace ser «humanos»). Esta es, tal y como afirma López Frías,

Una conceptuación más positiva del término «dignidad» que no se limita a la negatividad del impedir dañar, o a la autonomía, sino que se preocupa por aquello que las personas son $\mathrm{y}$, sobre todo, por el hecho de que, en verdad, lleguen a alcanzar esos modos de vida que tienen razones para valorar» (López Frías 2014, p. 209).

Por último, en su análisis sobre el perfeccionamiento humano, George Annas (2002) afirma que los sujetos resultantes de las intervenciones de mejora perfeccionadora no tendrán en cuenta la dignidad humana en términos de reco-

5 Concretamente, Kass habla de «dignidad básica» $\mathrm{y}$ «dignidad plena». 
nocimiento recíproco, pues estrictamente hablando, no serán humanos al uso, sino «otra cosa» que ha sido ampliamente denominada como ser post-humano y que, sabiéndose superior a nosotros, podría materializar la esclavización de nuestra especie (completo dominio de los seres humanos no mejorados por parte de los seres post-humanos): «la nueva especie, o 'post-humana', probablemente considerará inferiores a los humanos antiguos y 'normales', incluso salvajes, y aptos para la esclavitud o el sacrificio (Annas et al 2002, p. 162).

\section{III. ¿SIRVEN LAS NOCIONES DE IDENTIDAD Y DIGNIDAD HUMANAS COMO RAZÓN ÉTICA PARA RECHAZAR LA MEJORA HUMANA PERFECCIONADORA?}

La alusión a la identidad y dignidad humanas es una constante en el debate en torno a la legitimidad o no de la mejora humana biotecnológica en sentido no-terapéutico o perfeccionista, esto es, la búsqueda del perfeccionamiento de las capacidades humanas positivas y no la detección y eliminación de patologías. Ahora bien, teniendo en cuenta los avances biotecnológicos y los posibles beneficios que éstos pueden conllevar para la especie humana, ¿sirven las nociones de «identidad»y «dignidad» humanas para llevar a cabo un argumento ético-crítico en contra de la mejora humana perfeccionadora? Una breve respuesta a esta pregunta es: 1) Sí, siempre y cuando la mejora perfeccionadora de la que estamos hablando es una mejora de carácter radical; y 2) No, si la mejora perfeccionadora a la que se está aludiendo es una mejora de carácter moderado o relativo.

Antes de analizar las nociones de identidad y dignidad como argumento para rechazar el mejoramiento humano no-terapéutico, conviene definir a qué nos referimos cuando hablamos de mejora radical y a qué aludimos cuando hacemos lo propio de la mejora moderada o relativa. En su obra Truly human enhancement y, anteriormente, en su obra Humanity's end, Agar lleva a cabo una clasificación de las intervenciones de mejora humana que se aleja de lo que es habitual en el debate contemporáneo, a saber, diferencia dichas intervenciones según su grado de alteración y alcance, y no según su naturaleza o carácter (ser terapéutica o perfeccionadora). De este modo, establece la distinción entre mejora moderada - «la mejora de atributos y habilidades significativos a niveles cercanos de lo que es posible actualmente para los seres humanos» (Agar 2014, p. xi) - y mejora radical - «la mejora de atributos y habilidades significativos a niveles que superan en gran medida lo que es posible actualmente para los seres humanos» (Agar 2014, p. xi)- con el objetivo de rechazar ésta última. Aceptamos la gradualidad en las intervenciones de mejora, del mismo modo que aceptamos la distinción habitual entre mejora terapéutica y perfeccionadora. Sin embargo, rechazamos el modo en que el pensador neozelandés utiliza la noción de mejora radical. En todo momento Agar utiliza ésta para rechazar el perfeccionamiento humano no-terapéutico (potenciamiento de las habilidades 
humanas sin búsqueda manifiesta de la salud), probablemente como consecuencia de la identificación mejora perfeccionadora-mejora radical llevada a cabo por parte de diversos pensadores en base a los ideales y pretensiones posthumanistas. ${ }^{6}$ Sin embargo, entendemos que puede haber formas de mejora humana perfeccionadora que se alejen de los presupuestos del posthumanismo, como por ejemplo incrementar la esperanza de vida humana en 10 años o nuestro coeficiente intelectual (si es que en eso consistiera la inteligencia humana) en 10 puntos. De este modo, y a diferencia de Agar, nuestra noción de mejora humana moderada o, quizás mejor, relativa (para diferenciarnos de aquélla), se fundamenta sobre la aceptación de toda intervención tecnológica cuyo fin sea manifiestamente terapéutico, y de aquellas intervenciones con objetivos perfeccionadores o no-terapéuticos que no supongan una alteración radical de las características convencionales del ser humano. Como mantendremos a continuación, el problema no es el carácter de la intervención biotecnológica -como parece entender gran parte del espectro bioconservador-, esto es, si se trata de un perfeccionamiento terapéutico o no-terapéutico, sino el grado de la intervención (mejora relativa o mejora radical $^{7}$ ). Veamos ahora caso por caso, cómo afecta esta distinción de grados en las intervenciones de mejora al uso de las nociones de identidad y dignidad humana para rechazar la mejora perfeccionadora.

En lo que respecta a la afección de las denominadas respectivamente por Agar y Glannon «memoria autobiográfica» y «memoria episódica», y la autenticidad y responsabilidad de nuestros actos señaladas por Sandel, estamos parcialmente de acuerdo con la perspectiva reflejada por Nick Bostrom y Toby Ord (2006). Según éstos, cuando nacemos no tenemos ningún tipo de conocimiento o experiencia (tabla rasa), y es durante las etapas de infancia, adolescencia y adultez cuando llevamos a cabo de forma interna (con manifiesta influencia externa/ambiental) un proceso de mejora físico-cognitivomoral radical hasta alcanzar nuestra identidad personal como seres humanos plenamente desarrollados. Para ambos pensadores, en la identidad de un sujeto

6 Buena parte de los pensadores adheridos al movimiento posthumanista entienden que la mejora perfeccionadora es y debe ser siempre radical. Debido a esto, pensadores más cercanos al bioconservacionismo acaban por llevar a cabo una identificación entre mejora perfeccionadora y mejora radical.

7 Nuestra propuesta de mejora relativa no se fundamenta únicamente en el rechazo de un perfeccionamiento humano de carácter radical (como hace Agar), sino en la aceptación de las técnicas biomédicas de mejoramiento para objetivos no exclusivamente terapéuticos. A pesar de que Agar ve claramente la diferencia de grado existente entre una mejora moderada y una mejora radical, rechaza el perfeccionamiento en sentido no terapéutico, mientras que nuestra propuesta es aceptar tal perfeccionamiento a niveles que no supongan consecuencias fatales para aspectos fundamentales del individuo como son su identidad y su dignidad. 
completamente desarrollado (un adulto con una identidad definida) intervienen de forma indispensable pocas o ninguna experiencia de las primeras etapas de su vida, por lo que una mejora radical como la propuesta desde el sector de pensamiento posthumanista no tendría por qué eliminar la identidad del sujeto, al igual que las diversas fases y cambios de su vida natural no lo han hecho. La idea es que, si la mejora radical natural que el ser humano lleva a cabo a lo largo de su vida no conlleva la desaparición de nuestra identidad personal y la aparición de una nueva post-mejora, entonces, ¿una mejora radical de carácter artificial lo haría? En este punto nos comprometemos con la afirmación de que los seres humanos llevamos a cabo una mejora radical natural desde nuestra etapa de crecimiento más temprana, hasta alcanzar la adultez. Sin embargo, esta mejora radical de nuestros atributos corresponde a un proceso de evolución, o quizás mejor, desarrollo biológico de nuestra especie que se materializa a lo largo de décadas y de forma progresiva.

A nuestro entender, el problema con la mejora humana no-terapéutica no es relativo a su carácter artificial sino, más bien, que se pretende llevar a cabo una mejora de atributos o cualidades que al ser humano le podría llevar miles de años conseguir (si es que fuera posible) en un espacio corto de tiempo (casi de inmediato tras la intervención). Es este «salto» o fractura considerable (mejora radical) entre el ser humano pre-mejora y el ser humano pos-mejora (post-humano) el que podría acarrear los problemas antes expuestos. Es posible que muchas de nuestras experiencias y conexiones psicológicas correspondientes a las primeras etapas de nuestras vidas no tengan un rol fundamental en la formación de nuestra identidad personal (nuestros primeros recuerdos, experiencias que recordamos vagamente y sin un contexto claro), pero parece plausible y hasta razonable que el conglomerado de las experiencias «memorables» o «indispensables» de nuestra infancia, adolescencia y adultez (experiencias clave que nos han marcado o nos han hecho «ser» de un modo u otro) sí que tengan un rol importante. A través de todas esas conexiones sabemos cómo hemos llegado a ser lo que somos y cómo somos, por eso es importante la supervivencia de la memoria autobiográfica o episódica. Si pasamos de $\mathrm{H}$ (humano) a $\mathrm{H}^{+}$(posthumano) de un día para otro, no tendremos ese bagaje. Pasaríamos a tener toda una serie de cualidades que antes no teníamos y de las que no guardamos ningún tipo de conexión psicológica. Todos nuestros anteriores deseos, intereses e inquietudes dejarían de tener importancia. El nuevo sujeto tendrá otras prioridades, y probablemente otra identidad. Sin embargo, no ocurría lo mismo si la mejora perfeccionadora fuera moderada o relativa, esto es, que no superara los límites de lo que es actualmente posible para la humanidad. El problema no es ser más inteligente y fuerte o tener una vida más larga, sino pretender ser radicalmente más inteligente, fuerte y longevo en un espacio corto de tiempo, tanto como para suponer un «shock» irrepa- 
rable para nuestra identidad. Aunque nuestras habilidades físico-cognitivas o nuestra esperanza de vida fuera ampliada moderadamente, nuestras «nuevas aptitudes» no serían tan distintas a las anteriores como para suponer una brecha insalvable en nuestras experiencias indispensables y en la forma en que nos hacemos responsables de nuestros actos o logros.

En lo que respecta a la violación de la dignidad humana en términos de reconocimiento recíproco y respeto mutuo entre sujetos humanos, expresada por Habermas y González, ${ }^{8}$ la premisa de que una mejora perfeccionadora -como podría ser la expansión de nuestras habilidades cognitivas o físicas- afectará a aquélla de forma insalvable es presumiblemente errónea, al menos en parte. Si entendemos que, más allá de su naturaleza u objetivo, las intervenciones biotecnológicas de perfeccionamiento humano son susceptibles de gradualidad, una mejora perfeccionadora relativa, esto es, una mejora de nuestras cualidades positivas naturales que no supere con creces lo que un ser humano ha logrado (el límite actual del ser humano sería la línea infranqueable), no supondría problema alguno. 9

No parece probable que si un sujeto con habilidades cognitivas normales (dentro de la media) es perfeccionado hasta alcanzar las habilidades cognitivas del recientemente fallecido físico británico Stephen Hawking, o que un sujeto con unas habilidades físicas normales adquiera las habilidades de un deportista de élite como el velocista jamaicano Usain Bolt, viole el reconocimiento y respeto hacia los otros seres humanos como consecuencia de la intervención de mejora ni, de igual forma, que el resto de seres humanos rompan esa reciprocidad. De hecho, es habitual en la comunidad humana que sujetos con diferencias naturales significativas (no insalvables) en sus cualidades físicas, intelectuales e incluso vitales y morales convivan cordialmente e incluso mantengan relaciones estrechas sin que dé lugar a una «asimetría relacional» o a una falta de respeto del otro como miembro de la humanidad. De este modo, $\mathrm{y}$ aunque algunos seres humanos no estuvieran de acuerdo en someterse a

8 A diferencia de la concepción de «dignidad humana» en sentido relacional adoptada aquí, hay quien entiende -como Bostrom (2005) y (2008) - que la dignidad es una cualidad (gradual) que puede ser atribuida indistintamente a entidades humanas y no humanas (animales, entornos naturales, sociedades y civilizaciones), de modo que la mejora humana biotecnológica radical no reclama la protección de una «dignidad humana» en los términos anteriormente referidos, sino que lo que entendemos por dignidad acoja no sólo a la especie humana, sino a otras entidades, como podría ser la especie post-humana pretendida por el posthumanismo.

9 Otro supuesto a tratar sería si una mejora moderada afectaría a la autonomía o libertad de los sujetos en los términos establecidos por Habermas, especialmente en el caso del diagnóstico pre-implantatorio y la selección embrionaria, aunque limitar la mejora biotecnológica perfeccionadora a seres humanos con derecho jurídico a elegir sobre sus vidas (adultos capaces de elegir por sí mismos) podría superar esta traba. 
una intervención biotecnológica de perfeccionamiento por motivos culturales, religiosos o, simplemente, personales, no habría problemas de afección a la dignidad y el respeto humano en sentido relacional entre los seres humanos resultantes de una intervención relativa de mejora y aquéllos que han decidido no ser intervenidos. ${ }^{10}$

Sin embargo, una mejora perfeccionadora radical sí que supone un problema. Un ser humano convencional que ha sido perfeccionado hasta que sus características naturales superen ampliamente lo que es posible para un ser humano de forma natural (súper fuerza/resistencia, súper inteligencia, súper moralidad e inmortalidad), se diferencia estrictamente del resto de seres humanos. Tanto así que éste podría considerarse tan diferente a los seres humanos (físico-intelectual-moralmente superior o, simplemente, distinto en puntos esenciales a nivel de identificación con nuestra especie) como para que el reconocimiento y respeto recíproco entre él y los seres humanos convencionales deje de tener sentido. Quizás las consecuencias no sean tan «post-apocalípticas» como una guerra entre humanos y post-humanos, o la esclavización de los seres humanos convencionales a manos de los mejorados, tal y como manifiesta Annas (2002), pero todas las relaciones sociales, culturales, jurídicas e incluso morales de nuestra civilización habrían de ser reformuladas para acoger a la nueva realidad humana, que sería la de la convivencia entre los seres humanos biotecnológica y radicalmente mejorados, y los seres humanos «naturales». En cierto sentido, Bostrom (2005) estaba en lo cierto al reclamar la inclusión de la «dignidad posthumana» por parte de la «dignidad humana», porque en el última instancia y por el bien de ambas formas de vida (la de los mejorados y los naturales), el entendimiento, reconocimiento y respeto entre ambas partes se antoja esencial para su convivencia y coexistencia.

\section{CONCLUSIÓN}

Si bien es cierto que las nociones de identidad y dignidad humanas guardan en sí cierto halo de ambigüedad y equivocidad, en este artículo hemos adoptado la dupla «identidad numérica-narrativa» y la «dignidad relacional» como definiciones abiertamente aceptadas y utilizadas en el debate científicofilosófico contemporáneo en torno a la mejora humana biotecnológica. El ob-

10 En este punto nos alejamos de aquella perspectiva que entiende la mejora universal como una necesidad, esto es, que todos los seres humanos deberían ser mejorados para salvaguardar la igualdad entre sujetos. Una mejora humana relativa no supone un cambio entre sujetos tan extremo como para suponer problemas de igualdad añadidos a los ya existentes por la brecha socio-económica entre individuos, países y continentes. Entendemos que sería mucho grave tratar de obligar a cualquier ser humano a someterse a una intervención de mejora, pues en cierta medida, se estaría regresando a la vieja eugenesia y a sus métodos coercitivos. 
jetivo era mostrar cuáles podrían ser las consecuencias de una mejora humana de carácter no-terapéutico (perfeccionador) para ambas nociones, y dilucidar si es legítimo acudir a ellas para construir un argumento ético en contra de aquélla, tal y como viene haciendo gran parte del espectro bioconservador desde la emergencia de tecnologías biomédicas como la fecundación in vitro o los diagnósticos genéticos en el último tercio del siglo XX.

Tras el análisis de las consecuencias para la identidad y dignidad humanas con motivo de una mejora biotecnológica perfeccionadora propuestas por pensadores de renombre en el campo de la bioética como Habermas o Kass, entre otros, se ha llegado a dos conclusiones que, en esencia, es una misma: sólo una mejora no-terapéutica de carácter radical, esto es, un perfeccionamiento u optimización de las capacidades humanas naturales que supera con creces los límites alcanzados de forma natural por los seres humanos (como por ejemplo prolongar nuestra vida hasta alcanzar los 1000 años), podría afectar gravemente a la identidad y dignidad humanas en los términos sugeridos por la corriente de pensamiento bioconservadora, y por tanto, sólo en tal supuesto estas nociones podrían ser utilizadas en un argumento ético en contra de aquélla. Al mismo tiempo entendemos que, por el contrario, un mejoramiento biotecnológico de carácter relativo -aquél que no supera los límites naturales de la humanidadpodría ser una alternativa interesante de cara a un posible desarrollo científico de nuestras cualidades en términos no meramente terapéuticos, que supere y haga reformular los argumentos de los pensadores bioconservadores frente a la posibilidad de una mejora humana en sentido perfeccionador.

\section{REFERENCIAS BIBLIOGRÁFICAS}

AGAR, N. 2014: Truly human enhancement. A philosophical defense of limits. Cambridge: MIT Press.

ANDORNO, R. 1998: Bioética y dignidad de la persona. Madrid: Tecnos.

ANNAS, G et al. 2002: «Protecting the endangered human: toward an international treaty prohibiting cloning and inheritable alterations», American Journal of Law and Medicine, 28, pp. 151-178.

APARISI, Á. 2013: «El principio de la dignidad humana como fundamento de un bioderecho global», Cuadernos de bioética, 24/2, pp. 201-221.

BELLVER, V. 2012: «El debate sobre el mejoramiento humano y la dignidad humana: una crítica a Nick Bostrom», Teoría y Derecho. Revista de pensamiento jurídico, 11, pp. 82-93.

BOSTROM, N. 2005: «In defense of posthuman identity», Bioethics, 19/3, pp. 202-214.

BOSTROM, N y ORD, T. 2006: «The reversal test: eliminating Status Quo bias in applied ethics», Ethics, 116, pp. 656-679.

BOSTROM, N. 2008: «Dignity and Enhancement», en: Schulman A (ed.), Human 
dignity and bioethics: Essays commissioned by the president's council on bioethics. Washington D.C.: President's Council on Bioethics, pp. 173-207.

CABRERA, L. 2017: «Personas y seres humanos ¿distinction o identidad? », IUS ET SCIENTIA, 3/1, pp. 114-124.

CAMPS, V. 2002: «¿Qué hay de malo en la eugenesia?». Isegoría, 27, pp. 55-72.

CANTARINO, E. 2015: «Identidad y alteridad como constructos psicoeducativos y neurocognitivos», en: Ortega C; Richart, A; Páramo V y Ruíz C (eds.), El mejoramiento humano: avances, investigaciones y reflexiones éticas y politicas, Granada: Comares, pp. 807-818.

CINCUNEGUI, J. 2015: «Modelos de identidad. Hermenéutica, filosofía analítica y el cuasi-budismo de Derek Parfit». Nuevo Pensamiento, 5/6, pp. 1-51.

DEGRAZIA, D. 2005: «Enhancement Technologies and Human Identity». Journal of Medicine and Philosophy, 30, pp. 261-283.

DE MIGUEL BERIAIN, Í. 2018a: «Should human germ line editing be allowed? Some suggestions on the basis of the existing regulatory framework», Bioethics, 00, 1-7.

DE MIGUEL BERIAIN, Í. 2018b: «Problemas éticos y jurídicos que plantea la edición genética mediante CRISPR-Cas: un breve comentario», Genética médica y genómica, 0, pp. 1-3.

GLANNON, W. 2002: «Identity, prudential concern, and extended lives». Bioethics, 16/3, pp. 266-283.

GONZÁlEZ VALENZUELA, J. 2005: Genoma Humano y Dignidad Humana. Barcelona: Anthropos Editorial.

KANT, I. 2008: Metafísica de las costumbres. Madrid: Editorial Tecnos.

KASS, L. 2004: Life, liberty and the defense of dignity: the challenge for bioethics. New York: Encounter Books.

KASS, L. 2008: «Defending human dignity», en: Schulman, A (ed.), Human dignity and bioethics: Essays commissioned by the president's council on bioethics. Washington D.C.: President's Council on Bioethics, pp. 297-331.

LAING, J. 2009: «Los Derechos Humanos y la nueva eugenesia». SCIO, 4, pp. 65-80.

LAPORTA, F. 2013: «Identidad y derecho». Anuario de la Facultad de Derecho de la Universidad Autónoma de Madrid, 17, pp. 17-38.

LÓPEZ FRÍAS, F. 2014: «Dignidad y bioética: el caso de las intervenciones biotecnológicas de mejora de la especie». Thémata, 49, pp. 203-215.

PARFIT, D. 1983: «Identidad personal». Cuadernos de crítica, 25, pp. 5-36.

PARFIT, D. 2004: Razones y personas. Madrid: Machado Libros.

POSTIGO SOLANA, E y DÍAZ DE TERÁN, M. 2004: «Nueva eugenesia: la selección de embriones In Vitro», en: Ángela Aparisi y Jesús Ballesteros (eds.), Biotecnología, dignidad y derecho: bases para un diálogo, Navarra: EUNSA, pp. 79-113.

ROMEO CASABONA, C. 1994: El Derecho y la Bioética ante los límites de la vida 
humana. Madrid: Editorial Centro de Estudios Ramón Areces.

SAGOLS, L. 2010: «¿Es ética la eugenesia contemporánea?», Dilemata, 2/3, pp. 27-43.

SALLES, A. 2015: «La neurociencia y la identidad: un debate abierto», en: Ortega C; Richart, A; Páramo V y Ruíz C (eds.). El mejoramiento humano: avances, investigaciones y reflexiones éticas y políticas, Granada: Comares, pp. 57-66.

SANDEL, M. 2015: Contra la perfección. La ética en la era de la ingeniería genética. Barcelona: Marbot.

SPINELLA, L. 2012: «El sujeto de derecho a reivindicar: la Humanidad. La dignidad humana frente al patentamiento de la información genética humana», Revista Facultad de Derecho y Ciencias Políticas, 42/116, pp. 297-328.

Jesús PARRA es doctorando en Filosofía en la Universidad de Murcia

Linea de investigación: Éticas aplicadas.

Publicaciones recientes:

Parra Sáez, J. «El movimiento eugenésico estadounidense como clave del éxito de la eugenesia en el siglo XX y la posibilidad de su retorno en el siglo XXI» en Ágora: Papeles de Filosofia, 2018, № 37/2, pp. 123-148.

Parra Sáez, J. «¿Es la mejora humana biotecnológica la solución a los graves problemas medioambientales del planeta Tierra?» en Lecturas de nuestro tiempo, 2018, № 3, pp. 55-67.

Email: jesus.parra@um.es 
